\title{
Une approche du champ de la communication organisationnelle aux États-Unis
}

Bertrand Parent

\section{(2) OpenEdition}

1 Journals

Édition électronique

URL : http://journals.openedition.org/communicationorganisation/2958

DOI : 10.4000/communicationorganisation.2958

ISSN : $1775-3546$

Éditeur

Presses universitaires de Bordeaux

Édition imprimée

Date de publication : 2 octobre 2004

ISSN : 1168-5549

Référence électronique

Bertrand Parent, "Une approche du champ de la communication organisationnelle aux États-Unis », Communication et organisation [En ligne], 25 | 2004, mis en ligne le 10 octobre 2017, consulté le 19 avril 2019. URL : http://journals.openedition.org/communicationorganisation/2958 ; DOI : 10.4000/ communicationorganisation.2958

Ce document a été généré automatiquement le 19 avril 2019

(c) Presses universitaires de Bordeaux 


\title{
Une approche du champ de la communication organisationnelle aux États-Unis
}

\author{
Bertrand Parent
}

1 Des travaux sur l'usage rapproché des notions de «communication» et d'«organisation", m'ont amené à explorer le champ de la communication organisationnelle aux USA. Il s'agissait d'aller voir comment dans d'autres lieux et d'autres traditions scientifiques, ces deux termes pouvaient être employés et articulés. L'exploration de la littérature américaine utilisant centralement ces notions, conduit à rencontrer ce que certains auteurs, Linda Putnam ou Philipp Salem par exemple (entretiens 1999), considèrent comme un espace de recherche spécifique des "communication studies». Ce texte voudrait rendre compte de ce parcours dans la production de chercheurs supposés homologues à ceux du groupe Org \& Code la SFSIC, et proposer une analyse critique du champ scientifique ainsi identifié. Quels sont les principaux thèmes de recherche rencontrés, quelles sont les méthodes employées, quels sont les fondements théoriques des différentes approches et modes de problématisation repérés ? Les chercheurs français pourront trouver ici des éléments d'orientation dans la littérature anglo-saxonne (une partie) traitant de sujets proches de leurs préoccupations, éventuellement des pistes de travail, mais surtout, quelques éléments de comparaison avec leur propre réseau scientifique.

2 Cette analyse prolonge l'article de Valérie Carayol paru en $1998^{1}$. Elle s'appuie principalement sur le travail de $S f e z^{2}$

3 Le corpus étudié comprend plusieurs ouvrages de synthèse, des articles scientifiques et de très nombreux résumés d'articles, tirés de la base «ComAbstracs" du CIOS (Communication Institute Online Scholarship). Le matériel utilisé n'est pas exhaustif, mais couvre largement la production, sur prés de 20 ans, du champ académique de la communication organisationnelle aux USA. Les articles ont été publiés dans environ 70 revues scientifiques en communication. Ces documents sont cités ou référencés par les travaux sur l'état des recherches dans la discipline et les annuaires bibliographiques dont 
nous disposions. Ils contiennent tous, dans leur titre ou dans le corps du texte, l'expression "organizational communication ». Des entretiens effectués avec plusieurs auteurs se réclamant spécifiquement de ce champ de recherche ont complété l'analyse de contenu.

4 L'exposé comprend deux moments. Le premier présente le champ de la communication organisationnelle aux USA tel qu'il se donne à voir dans les articles de synthèse et les ouvrages généraux. Le deuxième tente, selon une approche plus critique, de dégager les fondements théoriques qui structurent cet espace de production de savoirs.

\section{Quelques caractéristiques du champ}

5 Un champ scientifique est un espace social particulier. On peut le caractériser par des objets de recherche spécifiques, des manières de poser les problèmes et des méthodes d'investigation propres, mais aussi, par des institutions représentatives, un droit d'entrée, des programmes de recherches et des laboratoires assurant son autonomie. L'histoire et la définition (légitime) d'un champ sont toujours l'objet d'une lutte entre membres. Cette lutte s'effectue aussi en rapport avec la dynamique d'autres champs ou sous-champs scientifiques (Bourdieu 2001). Ici, le champ américain de la communication organisationnelle sera caractérisé en confrontant les contenus de statuts d'associations professionnelles, de manuels scolaires, d'ouvrages et d'articles de synthèse présentant explicitement la dynamique du réseau. Leurs auteurs sont très cités dans le corpus retenu. Certains d'entre eux

Redding W.C, Tompkins P, Organizational Communication-Past and Present Tenses in Goldhaber \& Barnet, in Handbook of Organiszatuinal Communication, Norwood Ablex 1988 parlent même au nom de la prétendue communauté. Cette présentation reprend donc en partie, le point de vue dominant.

\section{Développement historique et définition}

7 Bien que de nombreux étudiants aient étudié depuis de nombreuses années la communication dans les organisations, le développement de a communication organisationnelle comme matière principale d'enseignement, avec un programme propre au sein de départements de communication des universités américaines, semble quant à lui, relativement récent. W. Charles Redding situe l'origine de ce champ dans la période où s'est accru l'intérêt pour le « discours entrepreneurial » (la langue, la rhétorique des affaires) et « la communication industrielle », ceci à l'aube de la Seconde Guerre Mondiale (Redding, 1985 ; Tompkins \& Redding, 1988) ${ }^{3}$.

Dès le début des années 1950, quelques étudiants des départements de communication des Northwestern University, Ohio State University, Purdue University et University of Southern California rédigent des mémoires sur la communication des entreprises industrielles. En 1952, le département Communication de Purdue met en place son "Centre de Recherche en Communication Industrielle». Des conférences annuelles rassemblent alors des chercheurs en communication et des spécialistes des sciences humaines. Des livres axés sur les "savoir-faire » et les outils de la communication destinés aux dirigeants d'entreprises sont publiés dés la fin des années 1950. 
9 L'objet de ce champ dépassera vite les préoccupations principalement managériales. En 1962, pour la première fois aux Etats-Unis, la communication organisationnelle ( organizational communication ») est enseignée comme matière principale par Edward Penson et Elizabeth Andersch dans l'Université d'Ohio.

10 Encore selon Redding, l'institutionnalisation de la communication organisationnelle dans l'université est signalée par divers événements entre 1967 et 1968, tels une conférence parrainée par la NASA ou la création de la division communication organisationnelle ("Organizational Communication ») au sein de l'International

11 Communication Association» (ICA). Selon cette association, le champ de la communication organisationnelle recouvre l'ensemble des savoirs théoriques ou appliqués, produits de l'analyse « du rôle de la communication dans le fonctionnement d'organisations complexes ». Cette définition se retrouve dans les statuts de la plupart des associations américaines en communication (ACA, ASCA.). De même, l'Academy of Management considère que le domaine spécifique de la division OCIS (Organizational Communication and Information System) concerne l'étude des aspects sociaux et comportementaux de la communication et des systèmes d'information dans et entre les organisations.

\section{Modes de problématisation}

12 Il existe plusieurs manières de décrire et présenter les différents points de vue en communication organisationnelle. Certains commentateurs en identifient de très nombreux, d'autres n'en distinguent que deux. La présentation suivante s'inspire des analyses proposées par Linda Putnam (1982), Philip Tompkins et Charles Redding (1988)4.

\section{L'approche fonctionnelle}

13 L'approche fonctionnelle consiste à comprendre la communication dans les organisations en décrivant la fonction et la nature des messages qui y circulent. De ce point de vue, on peut considérer la communication comme un processus complexe qui possède des fonctions d'organisation, de mise en relation et de changement. Ces capacités reviennent ici aux messages eux-mêmes. L'étude des réseaux (schémas des communication formelles ou informelles) et des canaux de communication (moyens de transmission), de la direction des messages (orientations verticale, horizontale...), de leur quantité et de leur distorsion (modification de la direction des messages au cours de la transmission) permet de déterminer le rôle de la communication à l'intérieur de l'organisation ${ }^{5}$. Cette approche emprunte principalement aux travaux du « Management Scientifique ${ }^{6}$ et à l'école des « Relations Humaines ».

\section{L'approche systémique}

14 L'approche systémique apparaît comme un outil précieux pour de nombreux chercheurs en organisation. Elle permet le renouvellement des études en sciences humaines et sociales par l'emprunt de concepts aux sciences de l'ingénieur, à la biologie, à la chimie, ou encore à la physique. Les biologistes L. Von Bertalanffy et J.G. Miller restent 
aujourd'hui encore, très connus aux USA pour leurs travaux. Ces auteurs ont inspiré de nombreuses recherches en sciences sociales.

Dans ce sens, des analogies de fonctionnement et des similitudes d'organisation sont supposées entre structures physiques, biologiques et sociales. Ainsi, avec la notion de système, défini comme «ensemble d'éléments interdépendants, organisés et autorégulé », là où « il est impossible de connaître les parties sans connaître le tout et de connaître le tout sans connaître les parties ", les chercheurs renouvellent leur approche du problème de la communication dans les « systèmes » sociaux.

16 La théorie des systèmes place en effet sous un éclairage particulier les processus de communication dans les organisations. Son utilisation en communication organisationnelle commence dans les années 1960. Le livre de Daniel Katz et Robert Kahn "The Social Psychology of Organisions» NY (1966) parait précurseur. Enthousiasmés par l'apport potentiel de cette approche, Richard Farace, Peter Monge, et Hamish Russell lui consacrent un ouvrage majeur, "Communicating and Organizing (1977).

Ils y développent l'idée, assez répandue aujourd'hui, selon laquelle «Communication is the process of organizing» («la communication est le processus par lequel on organise »).

Certains auteurs (Shockley-Zalabak, 1999) rappèlent cependant les limites épistémologiques de tels emprunts: l'usage de notions hors de leur contexte d'origine est rarement justifié et l'apport concernant le sens des communications parait généralement faible. Cependant, des efforts récents (Margaret Wheatley ${ }^{7}$, Peter Senge ${ }^{8}$ et Karl Weick ${ }^{9}$ ) renouvellent la théorie des systèmes en communication organisationnelle et cherchent à la rendre plus heuristique.

17 Les deux approches citées permettent l'analyse de la communication dans les organisations en décrivant la structure et la fonction des messages transmis. Même basées sur une conception de l'organisation comme système complexe, ces perspectives considèrent toujours la communication selon le schéma classique de l'envoi du message, d'un émetteur à un récepteur, par l'intermédiaire d'un canal. On parle de communication verticale, horizontale... On suppose des effets (qui dit quoi, à qui, comment, avec quels effets). On retrouve ici le modèle canonique de la communication, importé des sciences de l'ingénieur (Shannon) vers les sciences sociales.

Ce transfert, souvent légitimé par des études empiriques, réduit le problème de la communication à celui de la transmission des messages. Seule l'efficacité de la transmission suscite l'intérêt. Ces approches fournissent, directement ou indirectement, des outils pour l'action. ${ }^{10}$

\section{L'approche interprétative}

19 L'approche dite « interprétative » vient en contrepoint des perspectives plus rationnelles et formelles qui l'ont précédée et la côtoient. L'approche interprétative de la communication dans les organisations cherche peu à savoir «comment ça marche " ( II low and why il works?»), mais s'intéresse plutôt au sens même de la communication («What communication is?» L. Putnam, entretien 1999).

20 Il s'agit de savoir comment le fait organisationnel s'établit dans les interactions sociales. Ici, la structure et la fonction des messages semblent secondaires. La communication devient un processus de construction d'une communauté de sens ("shared realities»). 


\section{Le point de vue de Weick}

21 C'est avec l'ouvrage "The social psychology of organizing" (1979), que Karl Weick a contribué le plus au renouvellement du questionnement sur l'articulation entre organisation et communication. Pour lui, les

Poole MS, Another turn of the wheel: a return to systems theory in organizational communication, in Organizational communication and change conference, Austin, 1996

Monge P, Systems theory and research in the study ot organizational communication: the correspondence problem, in Human Communication Research N 8, 1982 organisations n'ont pas d'existence en dehors des interactions humaines ("through ongoing human interactions»). Ces interactions "enact» les environnements organisationnels. Chaque événement, chaque comportement n'a de sens que situé dans l'environnement organisationnel qu'il participe à construire. Dans cette perspective, les phénomènes organisationnels correspondent toujours à des processus de communication. Ainsi pour cet auteur, les organisations n'existent pas hors des interactions humaines, hors des interrelations entre membres et donc, hors de la communication. Il peut alors dire : "communication is the substance of organizing". On s'intéresse ici, moins aux aspects techniques ou pratiques des organisations qu'à leur dimension symbolique.

\section{De l'anthropologie à l'approche interprétative}

D'une manière générale, le concept de culture a connu un grand succès en sciences sociales aux USA. De nombreux courants et écoles caractérisent l'anthropologie culturelle américaine, mais c'est « l'anthropologie de la communication » avec Bateson et l'école de Palo Alto qui semble avoir inspiré le plus les sciences de la communication alors naissantes. En effet, empruntant elle-même aux travaux de Sapir, le premier aux USA à considérer la culture comme un système de communication interindividuelle ( le véritable lien de culture, ce sont les interactions individuelles ») et refusant tout discours essentialiste, cette école invite à l'analyse des significations partagées et des interactions symboliques. Ici, la communication n'est pas conçue comme une relation d'émetteur à récepteur, mais selon le modèle orchestral, résulte des interactions entre individus réunis pour jouer ensembles. Chacun participe à sa manière à l'exécution d'une partition invisible, la culture. Tout le travail des anthropologues de la communication consiste alors à analyser les interactions qui produisent, reproduisent et transforment les systèmes culturels d'échange.

Dans cette perspective, analyser la culture implique de porter une attention particulière aux langages, aux histoires, aux communications non verbales et à toutes les formes d'échanges symboliques qui s'effectuent dans l'organisation.

Celle-ci produit du lien social, des appartenances, suscite des adhésions (ou des rejets) à des normes et des valeurs qu'elle sécrète. La culture organisationnelle est alors pensée comme le résultat d'une articulation complexe entre ce qui est produit spécifiquement dans une organisation (règles, normes, procédures qui façonnent les comportements) et les cultures des groupes présents (identités socioprofessionnelles.).

Le vocabulaire anthropologique a permis aux chercheurs (mais aussi aux managers) de renouveler leurs analyses des organisations. Dans ce sens, "the Alla Conference on 
Interpretative Approaches to Organizational Communication » organisée par Linda Putnam et Michael Pacanowsky, semble avoir joué un rôle majeur. Les principales communications furent publiées dans le livre "Communication and Organizations: An Interpretative Approach » (1983). De même, l'article de Pacanowsky et O'Donnell-Trujillo ${ }^{50}$, a lui aussi largement contribué au développement du point de vue "interprétatiste» en communication organisationnelle.

\section{L'approche critique}

" les organisations ne sont pas des lieux neutres de production de sens et d'échange; au contraire, elles émergent dans des contextes de luttes entre groupes d'intérêts et des systèmes de représentation concurrents ». Pour Stanley Deetz (1992, 1995), auteur très cité en communication organisationnelle, il s'agit de défendre les individus de l'emprise de certaines formes d'organisation («the corporate colonization of the life world'). Selon lui, la société doit s'assurer que les entreprises ne captent pas dans le bien commun, plus que ce qu'elles y restituent sous la forme de produits, services ou impôts. Le modèle des "groupes d'intérêts multiples $»^{13}$ s'entend comme dispositif de lutte vis-à-vis de l'économie mondialisée et l'économisme.

32 Ainsi, pour un certain nombre de chercheurs, «sous les apparences de rationalité d'un monde de plus en plus modelé par la technologie et la science, se manifeste l'irrationalité d'un modèle d'organisation de la société qui, au lieu de libérer l'individu, l'asservit $»^{14}$. A la manière d'Habermas cherchant « la restauration des formes de communication dans un espace public élargi à l'ensemble de la société ${ }^{15}$, ces auteurs appellent de leurs voeux l'émergence de formes d'organisations plus démocratiques.

La perspective critique en communication organisationnelle est parfois considérée comme révolutionnaire ("revolutionary approach", "subversive approach", L. Putnam mondialisé. Le travail scientifique prend ainsi une dimension explicitement politique. Le chercheur dénonce les formes d'oppression et d'exploitation rencontrées dans les organisations.

trouverait ses fondements dans le mouvement « critique européen » (la théorie critique), conduit principalement par l'école de Francfort. De plus, les années 80 voient la remise en cause du modèle fordiste de répartition des richesses. Avec l'internationalisation du commerce et des échanges, la société découvre ou redécouvre la flexibilité, le chômage, la pauvreté. Ces éléments participent à l'émergence de l'approche critique en communication organisationnelle.

d'ailleurs parfois appelée «critica! interprétative perspective». Les chercheurs s'intéressent non seulement au fonctionnement des organisations (« le comment »), mais aussi aux raisons et aux causes de ce fonctionnement (« le pourquoi »).

Dans cette perspective, l'organisation est considérée principalement comme un système politique, un lieu où s'exercent des contrôles, où se produisent et reproduisent des rapports sociaux de domination. 
S. Deetz et M. Alvesson résument ainsi cette idée : «critical theory sees its work as a response to specific social conditions. Contemporary society as a result of science, industrialization, and communication/information technologies has developed positive capacities but also dangerous forms of domination. Critical theory describes western development as one where a progressive, instrumental modernism gradually eclipsed traditional society with fairly clear payoffs but also great costs. Something fundamental has gone awry and more technical, instrumental «solutions " will not fix it. $»^{16}$.

Bien qu'il existe d'autres manières de présenter les points de vue qui structurent le champ de la communication organisationnelle, les catégories exposés ci-dessus, sous les dénominations de "fonctionnel », « systémique », « interprétatif et " critique ", semblent couramment employées aux USA. L'ensemble des documents analysés, utilise cette terminologie. Si l'approche fonctionnelle parait avoir longtemps dominé les recherches, les travaux se sont progressivement tournés vers les concepts interprétatifs et critiques. En se dégageant des préoccupations managériales et s'ouvrant à la complexité des phénomènes de communication organisationnelle, ces dernières perspectives ont, à partir des années 80 , suscité de nombreuses recherches. Aujourd'hui, la distribution entre ces principaux modes de problématisation semble homogène.

\section{Méthodes de recherche}

Quatre modes de probléniatisation ont été distingués pour caractériser le champ de la communication organisationnelle aux USA. Si en pratique, de nombreuses recherches, par leur caractère transversal, atténuent les différences et estompent les frontières, il semble que trois groupes méthodologiques puissent aussi être identifiés. Ces différences se mesurent en comparant les recherches sur la culture organisationnelle.

L'approche fonctionnelle produit du savoir en comparant des formes de communication à des éléments du fonctionnement des organisations. On note par exemple des travaux qui croisent structure des systèmes d'information et performance organisationnelle. Les méthodes sont en général quantitatives et statistiques. Dans cette perspective, la culture organisationnelle est analysée à partir de variables mathématiques. Ces analyses restent assez limitées. En effet, on peut attendre des études qu'elles s'intéressent aux significations, aux représentations, aux symboles, aux échanges qui contribuent à la culture d'une organisation. Ces données subjectives ne peuvent être traitées statistiquement. La culture n'est pas fixé une fois pour toutes, mais s'élabore continuellement dans les relations. Dans ce sens, le pôle interprétatif en communication organisationnelle appréhende les phénomènes culturels par des méthodes qualitatives, permettant en situation, de mieux rendre compte de la subjectivité des acteurs sociaux. Ce qui compte pour les analystes, ce sont les représentations et les significations que les individus se font de la réalité sociale. On peut citer des méthodes du type "fantasy theme analysis » ou " narrative analysis » $\|^{17}$...

En terme de méthodes, la perspective critique se rapproche du pôle interprétatif, employant principalement des analyses qualitatives.

Cependant, les données qualitatives sont rapportées aux structures sociales dans lesquelles s'effectuent les échanges. Pour les chercheurs, une organisation n'est pas un espace neutre. Les relations de pouvoir, la distribution des rôles et des qualifications établissent des cadres sociaux qui déterminent la position des acteurs, et par là même 
orientent leurs représentations, leurs choix et leurs identités. La culture ne peut s'appréhender hors des cadres sociaux dans lesquelles s'effectuent les échanges.

On trouve donc une gamme relativement vaste des méthodes de recherche en communication organisationnelle aux USA. Cette gamme s'étend d'un pôle fonctionnel, proche des intérêts managériaux, à un pôle critique qui ne s'émeut pas d'un certain interventionnisme. La logique de recherche peut être hypothético-déductive ou inductive, mais les données sont plutôt d'ordre quantitatif d'une part, et qualitatif de l'autre.

\section{Thèmes de recherche courants}

41 Des approches et des concepts aux origines disciplinaires multiples, des méthodes de recherche variées conduisent à la construction d'objets très différents. Le champ de la communication organisationnelle aux USA comporte cependant des thématiques de recherche qui varient assez peu.

\section{Communication as a process : communication structure and function}

42 Il s'agit d'un domaine de recherche maintenant classique. Centrés sur l'étude des messages et des caractéristiques de leur transmission (formelle, non formelle, verbale, non verbale) les travaux s'intéressent aux fonctions de la communication dans les organisations.

\section{Information technology}

Le regain d'intérêt pour des travaux concernant les réseaux de communication et les pratiques de communication organisationnelle, s'explique en partie par le développement des nouvelles technologies de l'information et de la communication dans les entreprises. Dans le contexte d'émergence de l'économie de l'information, la communication de fàceà-face parait de plus en plus remplacée ou suppléée par des télécommunications. On parle de communication assistée par ordinateur ( computer mediated communication «).

Ainsi de nombreux chercheurs observent les effets de l'innovation technique en matière d'information et de communication dans les organisations.

Les conditions d'appropriation et d'usage de ces nouvelles technologies, leurs impacts sur les pratiques, sur l'organisation du travail et la communication interpersonnelle suscitent de nombreuses études. Le domaine semble même exprimer certaines velléités d'autonomie par rapport au champ de la communication organisationnelle. Une revue spécialisée est désormais éditée (The Journal of Computer Mediated Communication).

Les premières recherches évaluent l'impact des technologies sur la distribution du pouvoir dans l'organisation, sur l'autorité et les modes de prise de décision (Casbolt et Cherry 1983, Danowski et Edison 1983...). De nombreux autres travaux concernent l'évolution de la communication de face à face dans ce contexte de communication électronique (Rice 1990, Carton et Wellman 1995, Poole et Desanctis 1992...). Certains observent une évolution relativement paradoxale. Dans un sens, les organisations mettent en place des systèmes technique de gestion de l'information, et dans l'autre (Roger 1988), leurs membres recherchent en retour, toujours plus d'interactions de face à 
face sur le lieu de travail. D'autres auteurs (Gratz et Salem 1984) s'inquiètent du développement de la communication homme machine au détriment de la communication interpersonnelle. Weick ${ }^{18}$ précise, par exemple, en quoi les nouvelles technologies interfèrent dans les interactions humaines et perturbent la production de sens et de signification en situation.

\section{Superior-subordonate communication}

Le «leadership» est bien souvent considéré comme condition nécessaire au fonctionnement efficace des groupes et des organisations. L'aptitude des managers («superiors ») au commandement, apparaît comme une qualité utile à la mise en place d'un collectif de travail et à sa gestion.

Les premiers travaux sur le leadership et la communication entre managers et subordonnés apparaissent avec W.C Redding à l'université de Perdue et F.Jablin à l'université du Texas. Jablin définit alors ce type de communication comme "exchange of information and influence between ce-o organizational members, at least one of whom has formal authority to direct and evaluate the activities of other organizational members ".

Dans l'étude des phénomènes de communication organisationnelle, de nombreux chercheurs américains s'intéressent au niveau d'organisation définissant le groupe. Celuici constitue le contexte des interactions interindividuelles. Pour eux, la qualité des communications dans et entre les groupes, influence considérablement les performances de l'organisation. Les recherches portent sur les formes de relations établies entre dirigeants et subordonnés, analysent les processus de prise de décision («decision making) et de résolution de problème (" problem solving»). Certaines études discutent la formation des normes et règles ("rule-bound process based on normative expectations and role enacment " (Daniels 1997) qui déterminent le statut des individus dans l'équipe. D’autres, analysent la variété des performances ("effective») entre différents types de groupes (Hirokawa 1980). L'efficacité du réseau dépend selon Brilhart et Galanes (1989), de la propension des individus à se sentir responsables du devenir de leur collectif. La littérature concernant l'étude des groupes dans les organisations est importante et variée en communication organisationnelle. Décrivant les comportements de groupes («small-group experiences of individuals in organizations») ou de différentes formes de collectifs («long standing teams, project team, prefab group, self-managing team, task force group, focus group.) rencontrés dans les organisations, les études semblent s'orienter de plus en plus vers l'analyse des «teambased organization". Appelées aussi des "flat organization", ces nouvelles formes d'organisation du travail se caractérisent par une structure hiérarchique aplaties (le nombre de managers est faible) et un réseau d'équipes autonomes (« self managing team »). Comme le suggèrent Mulvey, Veiga, Elsass (1996), ce modèle d'organisation nécessite de grandes capacités communicationnelles ${ }^{19}$ et d'implication chez les membres du groupe. ${ }^{20}$

Gensen A., Small group communication : theory and praciice, Bellemont, 1991. Poole M, Decision development in small group, in Communication monograph 48. Katz, The effect ol group longevity on project communication and performance, in

Admistration Science Quaterly, 1982

\section{Organizational culture}

"Organizational culture» est un concept majeur du courant interprétatif en communication organisationnelle. Dans les années 80 , des chercheurs ont ainsi légitimé 
l'étude de la dimension culturelle et symbolique des organisations. Jusque là, les recherches s'intéressaient surtout aux fonctions et à la structure des communications.

Ce concept de culture a connu un tel succès, que l'ensemble des courants de recherche l'emploie désormais.

\section{Ethics, democracy in the workplace, globalization}

De nombreux travaux sur l'éthique et la démocratie dans le travail (forme démocratique d'organisation $\mathrm{du}$ travail) apparaissent dans le champ de la communication organisationnelle. Un sérieux mouvement de remise en cause du modèle d'organisation hiérarchique se développe actuellement aux USA. Pour certains, des formes démocratique ne peuvent émerger que dans des espaces où la communication est privilégiée, où l'échange de connaissances et l'apprentissage collectifs sont reconnus comme essentiel à la bonne marche de l'entreprise. Ainsi, au moment où se développe une critique de plus en plus radicale des responsabilités sociales et écologiques de l'entreprise, apparaissent les concepts de "workplace democracy", de travail coopératif, d'émancipation et de "self managed work learns $»^{21}$

\section{Première réflexion critique}

Jusqu'ici, à partir d'un certain nombre de traces (ouvrages généraux, manuels scolaires, articles, descriptifs de cours, statuts d'associations) nous avons proposé une médiation « spontanée » au champ de la communication organisationnelle aux USA. Maintenant, en s'appuyant sur les travaux de Lucien Sfez et en particulier l'ouvrage « Critique de la

Abercombie N. Hill S., Turner B., The dominant ideology thesis, London, Allen, 1980. Ferguson K., The feminist case against bureaucracy, Philadelphia, temple University Press, 1984.

Morrill C. et Thomas C, Organizational conflict management as disputing process: the problem ol social escalation ", in Human Communication Research 18, 1992. . communication ", nous tenterons une analyse plus critique des travaux américains.

61 Eisenberg E... Dialogue as democratic discourse, in Communication Yearbook 17, 1994. Mc Phee R., Four critical approaches to workplace power/control in organizational communication «, ICA, 1985

La théorie critique de la communication

Le travail de Sfez a fortement contribué à la théorie (critique) de la communication. Située parfois au fondement d'un des principaux paradigmes de la communication, elle est apparue relativement récemment dans l'histoire des théories de la communication (les années 1960).

Elle fut élaborée au moment où, pour l'auteur, triomphe la «technique » dans les sociétés occidentales. Elle s'établit contre la communication devenue le nouveau mythe, la nouvelle religion. Il ne s'agit pas ici de décrire l'argumentation de Sfez, mais d'extraire de son travail des outils d'analyse du champ de la communication organisationnelle aux USA (telle qu'il se donne à voir).

Dans son ouvrage, Sfez décrit trois modèles de la communication: la communication comme représentation, la communication comme expression et la communication comme "tautisme ». Pour lui, les deux premières définitions de la communication vont de soi. Elles correspondent aux deux paradigmes classiques de la communication: le 
paradigme « linéaire des effets » et le paradigme « circulaire de l'interaction ». Ces deux paradigmes se déduiraient des premières définitions de la communication la communication comme communion dans un premier sens religieux au XIV ${ }^{e}$ siècle, auquel s'ajoute au XVIII ${ }^{\mathrm{e}}$ siècle le sens des ingénieurs, celui de transmission. L'ensemble des théories de la communication ne serait alors que la reformulation, dans des époques différentes (c'est-à-dire à des épistémologies et à des états de la technique différents), de ces premières définitions. Sfez décrit là, ce qu'il pense être les sciences «normales » de la communication, structurées autour de l'opposition nécessaire entre expression et représentation, communion et transmission, circulation et contrôle, théorie circulaire et théorie linéaire., symbole et technique. Sfez propose une troisième définition: la communication comme "tautisme» (néologisme qui condense totalité, autisme et tautologie), où « la communication se fait ici de soi à soi-même, mais dans un soi dilué dans un tout ». Cette définition ne va pas de soi pour Sfez. Elle apparaît au moment « où la machine créée par l'homme devient son propre créateur ", « où l'artefact est un effet qui se veut cause ». L'auteur tentera tout le long de son livre de critiquer cette nouvelle forme de communication. Pour la déconstruire, il cherchera à montrer en quoi elle constitue une confusion, inspirée par le "vieux rêve du double» et les travaux en intelligence artificielle: «on prend le représenter pour l'exprimer ou l'exprimer pour le représenter ». Contre cette percée dans les sciences sociales et les SIC, Sfez avec d'autres (Baudrillard, Legendre.) proposera la théorie critique de la communication. Nous disposons ainsi de trois modèles de la communication (communication comme expression, communication comme représentation et communication comme «tautisme ») et de trois paradigmes déduits de ces trois modèles. En effet, le paradigme « linéaire des effets » correspond à la communication-représentation et le paradigme « circulaire de l'interaction » à la communication-expression. Le paradigme critique est construit contre le modèle de la communication tautologique.

\section{La communication organisationnelle comme approche communi-cationnelle des organisations}

66 Appliquée à la communication organisationnelle aux USA, la proposition critique de Sfez semble très éclairante. En effet, comment ne pas retrouver dans la représentation proposée, les trois paradigmes de la communication explicités plus haut. En acceptant la catégorisation américaine du champ (fonctionnelle, systémique, interprétative, critique), on retrouve en soubassement les trois paradigmes cités.

En ce sens, la communication organisationnelle apparait d'abord comme une approche communicationnelle classique des phénomènes organisationnels: les «communication studies" s'intéressent aux phénomènes de communication; ces phénomènes sont centraux dans les organisations; d'où la communication organisationnelle comme approche communicationnelle (à partir des théories classiques de la communication) des organisations.

Le paradigme linéaire des effets

69 Avec l'approche dite fonctionnelle, on retrouve le modèle linéaire de la communication et la métaphore de l'organisation comme machine. Un message est transmis par un canal, d'un émetteur à un récepteur. La qualité de la transmission préoccupe en premier lieu (limitation du bruit). Il s'agit de la vision des ingénieurs en télécommunications, mais 
aussi celle des managers. Le paradigme du contrôle fonde cette perspective disent les auteurs situés dans celui de la circulation.

Pour Sfez, la communication est alors représentation. Il y a « extériorité et atomisation des éléments ». Le message est distinct de l'émetteur et du récepteur. Les sujets sont distincts l'un de l'autre. Un message est communiqué par l'intermédiaire d'une technique de communication.

71 Les situations sont représentées selon le mode déterministe de la causalité linéaire. La communication apparaît comme une variable de l'organisation, mesurable quantitativement. Les rapports entre les individus sont pensés sur le même mode: la réception des messages entre « supérieur et subordonné » suscite l'intérêt.

L'approche systémique constitue la charnière ou le passage vers l'autre paradigme de la communication. Dans les premiers temps, les textes présentent cette perspective de manière relativement simplifiée, voire simpliste.

La métaphore dominante devient celle de l'organisme. Y circulent des messages, envoyés d'un émetteur à un récepteur, sur le modèle du système nerveux. L'environnement ou le contexte de la communication se complexifient et de nouvelles variables (culture, désir.) interviennent. Le schéma linéaire de la communication reste prégnant, mais on commence à parler de rétroaction. La systémique est d'abord employée comme un outil de rationalisation des situations devenues plus complexes. La logique des premiers ingénieurs domine encore, et l'efficacité de la transmission motive les recherches. Avec l'approche interprétative, le passage à l'autre registre de la communication a lieu.

En empruntant principalement à la cybernétique, à l'interactionnisme et à l'anthropologie culturelle, la communication organisationnelle adopte complètement l'hypothèse systémique. La communication s'effectue maintenant à l'intérieur même du système, dans l'organisme social, qu'elle contribue à produire. Le sujet organique, plongé dans son environnement, ne peut se définir sans lui et réciproquement. 11 n'y a plus envoi par un sujet émetteur d'un message calculable à un objet récepteur. "La communication est alors insertion d'un sujet complexe dans un environnement lui-même complexe. » Le sujet fait partie de l'environnement et l'environnement fait partie du sujet. La logique est celle de la causalité circulaire. On se situe dans le registre de l'expression (j'exprime le monde qui m'exprime) dirait Sfez, c'est-à-dire le paradigme de la circulation. Les auteurs conçoivent en effet l'organisation comme le système complexe qui exprime ses membres autant qu'il est exprimé par eux. L'organisation s'apparente à un système culturel ou politique, qui produit, autant qu'il est produit par les interactions entre ses membres. La communication apparaît comme le lieu même de ces interactions.

\section{Le paradigme critique}

76 A y regarder de près, l'approche critique dans le champ de la communication organisationnelle aux USA ne semble pas correspondre exactement au paradigme critique tel qu'il a été défini plus haut.

Partageant sans doute la même crainte, héritée de l'école de Francfort, vis-à-vis de la raison instrumentale et $\mathrm{du}$ mouvement de rationalisation technique du monde, l'approche américaine semble cependant moins préoccupée par le problème de la 
communication instituée comme nouveau mythe de la conciliation universelle, que par les formes pratiques d'exercice de la domination dans les organisations. La démocratie ne se renforce pas obligatoirement avec l'apparition des nouveaux modes de production et le développement des nouvelles techniques de communication. La communication et les techniques de communication contribuent aussi au renouvellement des formes de violence symbolique et peuvent apparaitre ainsi comme des instruments de domination (" gestion symbolique »).

78 Au regard des travaux de Sfez, le champ de la communication organisationnelle aux USA apparait structuré selon l'opposition constitutive de la notion de communication: communion et transmission, expression et représentation, symbole et technique.

\section{Pour conclure}

Le domaine d'étude qui caractérise la communication organisationnelle semble assez large : des sujets et des problématiques très diverses font l'objet de travaux. En fonction des fondements ou des emprunts théoriques, il a été possible de décrire plusieurs approches en communication organisationnelle : les approches fonctionnelle, systémique, interprétative et critique. Ces approches renvoient à des hypothèses théoriques précises, qui déterminent des méthodologies de recherche particulières. De nombreuses problématiques restent cependant transversales et contribuent à l'ensemble des points de vue cités.

Comme l'indique G. Cheney (entretien 1999), les travaux s'orientent aujourd'hui vers l'étude des nouvelles formes d'organisations ("self organizing systems»), des cultures organisationnelles, des discours sur la globalisation, la démocratie dans le travail et les nouvelles technologies.

81 Si la communication organisationnelle semble dans sa construction avoir emprunté à de nombreuses autres disciplines, un effort épistémologique voit actuellement le jour aux USA. Le réseau constitué par le pôle interprétatif/critique (L.Putnam, P.Salem, G.Cheney, E.Eisenberg, P.Riley, S.Deetz, D.Mumby et M.Pacanowsky..) fait aujourd'hui mentir Philip Salem qui, en 1976, affirmait que la communication organisationnelle était athéorique. Aujourd'hui, les articles possèdent un contenu théorique beaucoup plus important.

Des concepts propres apparaissent " organizational culture ", " communication effectiveness ", "speech act theory». On dépasse les approches empiriques qui cherchent à résoudre à court terme des problèmes rencontrés dans les organisations. Le mouvement semble celui de l'intégration vers un paradigme précis ( It deals with the way both communication and organization constitute one another an intersect to form both the basis of organizing and the notion of communicating. ", L. Putnam, entretien 1999).

A cette étape de l'analyse, nous découvrons avec la communication organisationnelle aux USA, ce que Sfez a appelé par ailleurs «la science traditionnelle de la communication » des organisations, distribuée de manière classique entre un pôle fonctionnel et un pôle critique. La Communication Organisationnelle peut alors se comprendre comme un champ de recherche constitué en réponse à des préoccupations managériales et sur l'évidence de phénomènes sociaux ou techniques (Communication, Information), repérés dans les organisations et les entreprises. Ce champ émergent a alors suivi les évolutions majeures de la discipline de « tutelle » : interdisciplinarité, constructivisme., adoption du 
paradigme critique en opposition au paradigme fonctionnel... Il s'est ainsi étendu et complexifié, agrégeant des recherches de plus en plus théoriques.

\section{BIBLIOGRAPHIE}

OUVRAGES GENERAUX

BOURDIEU P, Science de la science et réflexivité. Raison d'agir, 2001

CONRAD C, Strategic Organizational Communication : Toward the 21st Century, 4th ed. Ft. Worth, Harcourt and Brace, 1998

DANIEL T, SPIKER B, PAPA M, Perspectives on Organizational Communication, Fourth Edition, McGraw HilL, 1997.

DEETZ S, Democracy in an age of corporate colonization : Developments in communication and the politics of everyday life, State University of New York Press, 1992

DEETZ S, Communication Yearbook 16, SAGE, 1993.

DEETZ S, Transforming communication, transforming business : Building responsive and responsible workplaces, Hampton Press 1995

FARACE R, MONGE P, RUSSEL H, Communicating and organizing, Mass Adisson-Wesley, 1977

KATZ D, KAHN R, The social psychology of organizations, Willey \& Sons, 1966

MORGAN G, Images de l'organisation, Les Presses de l'Université Laval, Editions Eska, 1989.

PUTNAM L, PACANOWSKY M (eds), Communication and Organization, Sage, 1983

SHOKLEY-ZALABAK P, Fundamentals of Organizational Communication, Fourth Edition, Longman, 1999.

WEICK K, The social psychology of organizing, Mass Addisson-Wesley, 1979

Articles en texte intégral :

BENSON T, « Communication Research and Theory Network » in CRTNET, Number 151, 1988.

BORMANN E, « Empowering as a heuristic concept in organizational communication » in Communication Yearbook 11, 1988.

BRILHART J, GALANES G, «Effective Group Decisions » in William C Brown Publishers, 1989

CASBOLT D, CHERRY J, « The decentralization of an organizarion's information center : organizationnal change issues and answers, ICA 1983

CHENEY G, " The rhetoric of identification and the study of organizational communication », $m$ Quarterly Journal of Speech 69, 198.3.

CHENEY G, MUMBY D, STOHL C, HARRISON T. M., « Communication and Organizational Democracy », in Electronic Journal of Communication, Volume 8 Number 1, 1998.

CONRAD C, « Communication in Conflict : Style - Strategy Relationships », in Communication Monographs 58, 1991. 
DANOWSKI J, EDISON-SWIFT P, « Crisis effects on intraorganizational computer-based communication », in Communication Research 12, 1985

Deetz S, «Critical interpretative research in organizational communication », in Western Journal of Speech Communication 46, 1982

FORD J, « Organizational Change as Shifting Conversations », Ohio State University, 1998.

GARTON L, WELLMAN B, « Social impacts of electronic mail in organizations : A review of the research literature » in Communication yearbook 18, Sage 1995

GRATZ R, SALEM P, « Technology and the crisis of self, in Communication Quarterly 32, 1984

HACKER K, «Predictors of communication satisfaction with the use of CMC as a tool of interuniversity discussion and learning », ICA, 1992.

HIROKAWA R, « A Comparative Analysis of Communication Patterns Within Effective and Ineffective Decision-Making Groups », in Communication Monographs 47, 1980

KLING R, « Organizational Analysis in Computer Science » in The Information Society, 9(2), 1993.

LOPERFIDO A, « Electronic mail as a media choice for managers » in Electronic Journal of Communication, Volume 3 Number 2, 1993.

MARSHALL J, « Viewing organizational communication from a feminist perspective », in Communication Yearbook 16, 1993.

METZLER M, «Organizations, Democracy and the Public Sphere » in Electronic Journal of Communication, Volume 8 Number 1, 1998.

MONGE P, FARACE R, EISENBERG E, MILLER K, WHITE L, « The process of studying process in organizational communication ", in Journal of Communication 34, 1984

MUMBY D, STHOL C, « Power and discourse in organization studies : absence and the dialectic of control », in Discourse U Society 2, 1991

MULVEY P, VEIGA, J, ELSASS P, « When teammates raise the white flag », in Academy of Management Executive 10, 1996

POOLE M, DESANCTIS G, « Microlevel structuration in computer-supported group decision making », in Human Communication Research 19, 1992

PUTNAM L, « Paradigms for organizational communication research : an overview and synthesis », in Western Journal of Speech Communication 46, 1982

PUTNAM L, CHENEY G, « Organizational communication : hisorical development and future directions ", in Speech Communication in the 20th Century, T BENSON, Southern Illinois University Press, 1995

RICE R, « Computer-mediated communication system network data : Theoretical concerns and empirical examples » in International Journal of Man-Machine Studic, 32, 1990.

RICHETTO G, « Organizational communication theory and research : an overview », in Communication Yearbook 1, 1977Rogers E, «Information technologies : How organizations are changing », in Goldhaber c> Barnett (Eds.), Handbook of organizational communication, Ablex 1988.

SWENSON LEPPER T, « Ethical Sensitivity, Cognitive Mapping, and Organizational Communication : a different approach to studying ethics in Organizations » in Electronic Journal of Communication, Volume 6 Number 4, 1996. 
WATKINS ALLEN M, GOTCHER M, SEIBERT J, « A Decade of Organizational Communication Research : Journal Articales 1980-1991», in Communication Yearbook 16, 1993.

WERT-GRAY S., CENTER C, BRASHER D. E., MEYERSR, « Research Topics and Methodological Orientations in Organizational Communication : A Decade in Review » in Communication Studies 42, 1991.

WILLIAM MAULE R, « Infrastructure Issues in Computer-Mediated-Communication » in Electronic Journal of Communication, Volume 3 Number 2, 1993.

Résumés d'articles scientifiques

L'ensemble des résumés d'articles publiés, dés les années 70, dans près de 73 revues scientifiques américaines en Communication et référencés par la base de données « ComAbstracts » du CIOS. Tous les résumés contenant l'expression « organizational communication » ont été sélectionnés (240). http ://

Compte rendu de conférences

Salem $\mathrm{P}$, « Organizational communication and change conference, challenge of the next century », ICA, 1995.

Statuts d'associations de communication

ICA (international Communication Association)

NSCA (National Speech Communication Association)

AM (Academy of Management)

CIOS (Communication Institute lor Online Scholarship) http ://

Entretiens Linda Putnam (Texas AM University) George Cheney (University of Montana) Philip Salem (Southern Texas University)

\section{NOTES}

1. Carayol V., La communication organisationnelle en perspective : pistes nord américaines in $L a$ Communication des entreprises et des organisations, Christian Le Moënne, PUR, 1998

2. Sfez L., Critique de la communication, Seuil 1988

3. Redding W.C, Stumbling toward identity : the emergence ot organizational communication as a field ot study, in Organizational Communication. Mc Phee \& P. Tompkins ed., Sage Publications, 1985.

4. Putnam I.., Paradigms tor Organizational Communication Research: an overview and synthesis, in Western Journal Communication 46, 1983.

5. Axley SR. Managerial and organizational communication in terms of the conduit metaphor, in Academy of management review 9, 1984

6. Redding W.C. et Tompkins P., Translating organizational theory : symbolism over substance, in Handbook of Organizational Communication, Sage Publications, 1988. Tompkins PK, The function of human communication in organization, in Handbook of rhetorical and communication theory, Boston. Allyn \& Bacon, 1984

7. Wheatley M., Leadership and the new science, San Francisco, Barett-Koehler, 1992

8. Senge P.,The fifth discipline, New York, Currcncy Douhlebay, 1990

9. Weick K., Sensemaking in organization, Thousand Ooaks, Sage, 199.S 
10. Phillips DC., Postpositisistic science : myths end realities, in The paradigm dialog, Newbury Park, SAGH, 1V90

11. M. Pacanowsky et N.O'Donnell-Trujillo, Organizational Communication as cultural performance, in Communication Monographs 50, 1983Mumby 1.

12. Mumby 1), Narrative and social control, Newbury Park, Sage, 1993).

13. Deetz S, Transforming Communication, transforming Business, Hampion Press 1998.

14. Mattelart A et M, Histoire des théories de la communication, La découverte 1997.

15. idem

16. Deetz S. et Alvesson M., Critical Theory and Postmodernism Approaches to Organizational Studies, in Handbook of Organization Studies. 1996.

17. Kosh S, Deetz S, Metaphor analysis of social reality of organisation. Alta Conference, ICA.1981.

18. Weick $\mathrm{K}$, Cosmos vs Chaos: Sense and Nonsense in Electronic Contexts, in Organizational Dynamics 14. 1985.

19. Jablin F, Superior-Subordinate Communication : The State of the Art, in Psychological bulletin 8 6, 1479 .

20. Godykunst W., Yoon Y., Influence of individualism-collectivism on perceptions of communication in ingroup and outgroup relationships, in Communication monograph 54, 19X7.

21. Mumby, Stohl C, Power and discourse in organization studies : absence and the dialectic of control, in Discourse and Society vol 2, 1991.

\section{RÉSUMÉS}

L'article propose une analyse critique du champ de la communication organisationnelle aux USA. À partir d'une revue de la littérature produite sur prés de 20 ans, le texte présente dans un premier temps, les principales caractéristiques du champ de recherche : conditions historiques d'émergence, modes de problématisation, thématiques de recherche, méthodes. Dans un deuxième temps, en mobilisant les travaux de Sfez, l'analyse tente de dégager les fondements épistémologiques des recherches identifiées. Ainsi, au terme de ce parcours, la communication organisationnelle aux USA apparaît comme une approche «communicationnelle » classique des organisations. Fondées sur l'opposition constitutive de la notion de communication (communion et transmission), les recherches se distribuent de manière classique entre un pôle fonctionnel et un pôle critique, mais convergent aujourd'hui vers un paradigme de la médiation, où communication et organisation sont deux notions considérées comme synonymes.

This article proposes a critical analysis of the field of organizational communication in the United States. Through a review of the literature produced during the last 20 years, the text presents firstly the major features of the research field : historical conditions of emergence, form of problematics, themes of research, methods... Secondly, using Sfez's work, the analysis tries to draw the epistemologica! fundamental of the research studied. Thus, this work leads to the conclusion that organizational communication in the United States is nothing else but a traditional « communicational » approach of organizations. Based on the constituent opposition of the communication notion (communion and transmission), research are divided between two approaches, one fonctionnai and the other critical, but they are converging today towards the paradigm of mediation, in which communication and organization are two notions considered as synonymous. 


\section{AUTEUR}

\section{BERTRAND PARENT}

Après des études de biologie, Bertrand Parent s'est tourné vers les sciences sociales et la recherche. A.T.E.R. en S.I.C. à l'université de Rennes 2 , sa thèse porte sur le rapport entre communication, information et processus organisationnels. Il par ailleurs conduit une étude critique sur le développement en France des recherches en communication organisationnelle, à partir des travaux américains dans le domaine. 